\title{
Design and Simulation of Uniform Magnetic Field
}

\author{
A. Mukhamedgali and Z. B. Rakisheva
}

\begin{abstract}
This article describes the design, construction and calculation of the mathematical model of a homogeneous magnetic field, considering for this Helmholtz coil. Helmholtz coils consist of a pair of coils along one axis parallel to each other. By varying the distance, size, winding of these coils, it is enough to simulate a homogeneous magnetic field that simulates the real Earth's magnetic field in orbit. Circular and square coils, both with square cross section, are considered. Practical considerations such as wire selection, wire-wrapping efficiency, wire bending radius, choice of power supply, and inductance and time response are included. Numerical calculations of the magnetic flux density, the values of the magnetic field strength vectors in various directions are presented. The main goal of creating this simulator is to test the development of algorithms for orientation and control of the spacecraft, to improve the functioning of the components of the satellite in the Earth's orbit
\end{abstract}

Index Terms - Coil system, Helmholtz coils, Magnetic field, uniform field.

\section{INTRODUCTION}

$\mathrm{A}$ $\mathrm{T}$ THE initial stages of development and design of the spacecraft (spacecraft), a hierarchical development of all functioning satellite subsystems is assumed. One of the most important service subsystems is the satellite attitude control system. To determine the orientation, various sensors, such as stellar, solar and magnetic sensors, can be used.

With the use of systems for determining the orientation based on the functioning of a magnetic sensor integrated into the onboard platform of a spacecraft, it is proposed to obtain these vectors of the Earth's magnetic field strength.

To test the operation of the designed control systems for spacecraft orientation, testing is conducted in laboratory conditions using various stands, simulators with which the conditions of outer space are simulated.

For this purpose, it is supposed in the laboratory conditions to create the imitation conditions of the geomagnetic field as much as it affects the onboard equipment and components of the satellite and the adequate functionality of the control algorithms for control and orientation of the spacecraft.

A. MUKHAMEDGALI, is with Dept. of Mechanics and Mathematics of Kazakh National University named after al-Farabi, Almaty, Republic of Kazakhstan, (e-mail: adilmukhamedgali@gmail.com).

Z. B. RAKISHEVA, is with Dept. of Mechanics and Mathematics of Kazakh National University named after al-Farabi, Almaty, Republic of Kazakhstan, (e-mail: zaure.ra@gmail.com ).

Manuscript received May 14, 2018; accepted October 20, 2018.

DOI: $10.17694 /$ bajece. 475537
There is a need to test and verify the developed algorithms for controlling a satellite with a magnetic orientation system in simulated conditions before it is put into orbit in order to minimize the risk of fail-safety of space borne space vehicles. For this goal in this paper, we are traditional Helmholtz coils design. One device capable of generating a uniform magnetic field is the Helmholtz coil [1]. The Helmholtz coil is named after Hermann von Helmholtz (1821-1894), a German scientist and philosopher known for his contributions to electrodynamics, mathematics, and meteorology among many other sciences [1].

The system of solenoid coils or Helmholtz coils [2-4] is often used for generating homogeneous field and calibration magnetic fields sensors. General analysis of the magnetic field in Helmholtz coils and has been presented in [4-7]. However, it is noticed that the homogeneous region of magnetic field in As shown in Fig. 1, a Helmholtz coil consists of a pair of coils parallel to each other where each coil consists of number $\mathrm{N}$ wrappings. A magnetic field B results when charge is in motion, i.e. as current I passes through the coil pair.

\section{THEORETICAL DESCRIPTION}

The Helmholtz coils illustrated in Fig.1 consist of two circular or square coils of the radius $\mathrm{R}$ or diameter $\mathrm{D}$, arranged at a distance $\mathrm{R}$ or $\mathrm{D}$ from each other. The coils with current source are generating a homogeneous field [8]. According of the law of Bio-Savart-Laplace, the resulting of homogeneous field of two coils is equal to the vector sum of the fields generating by one coil.

The homogeneous field by one coil can be calculating according to (1):

$$
B(z)=\frac{\mu_{0} N I R^{2}}{\left.2\left(R^{2}+(z-h)^{2}\right)\right)^{2 / 3}}
$$

where:

$\mu_{0}$ - the magnetic permeability of vacuum, $\mathrm{H} / \mathrm{m}$;

$N$ - number of turns of each coil-;

$I$ - Current in the coils, A;

$z$ - the axis which generating of magnetic field, m;

$R$ - radius of coils, m;

$h$ - distance of the coil center from the beginning of the coil center coordinates, $\mathrm{m}$.

"This article was presented during the International conference Alternative Energy Sources, Materials \& Technologies (AESMT'18), 14 - 15 May, 2018 in Plovdiv, Bulgaria" 


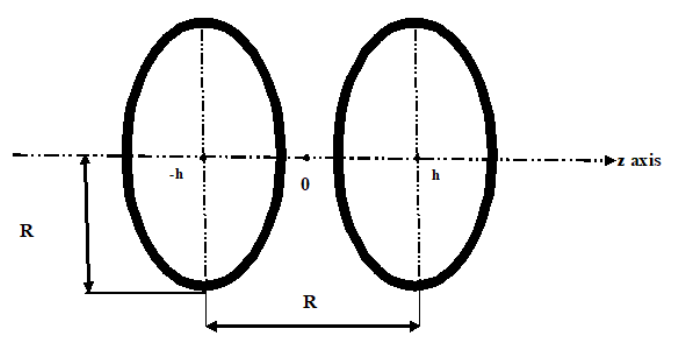

Fig.1. Geometric pattern of coils

Axial field of two coils can be calculated by formula:

$$
\begin{aligned}
& B(z)=\frac{\mu_{0} N I R^{2}}{2}\left\{\left[R^{2}+(z+h)^{2}\right]^{-\frac{3}{2}}\right. \\
& \left.+\left[R^{2}+(z-h)^{2}\right]^{-\frac{3}{2}}\right\}
\end{aligned}
$$

For calculating of Helmholtz, coils take the following form:

$$
\begin{aligned}
& h=\frac{R}{2}, \\
& B(z)=\frac{\mu_{0} N I R^{2}}{2}\left(\left[R^{2}+\left(z+\frac{R}{2}\right)^{2}\right]^{-\frac{3}{2}}\right. \\
& \left.+\left[R^{2}+\left(z-\frac{R}{2}\right)^{2}\right]^{-\frac{3}{2}}\right\}
\end{aligned}
$$

Consider the successive derivatives of for estimate the homogeneity of the field in center of the coil system. A higher order of the first non-zero derivative at this point provides better homogeneity of the field in its vicinity[2].

Equation (3) can be expanded in a Taylor series in the variable $\mathrm{z}$ near zero:

$$
\begin{aligned}
& B(z)=B(0)+\frac{1}{2} B^{(2)}(0) z^{2} \\
& +\frac{1}{24} B^{(4)}(0) z^{4}+\frac{1}{720} B^{(6)}(0) z^{6}+\ldots
\end{aligned}
$$

Since the coils according to the Fig. 1 are located at a distance of the radius from each other, the second derivative in the central system becomes zero. Then the change in the field $\Delta \mathrm{B}$ when moving from the point $\mathrm{z}=0$ is given by (5) and has a fourth order of smallness:

$$
\Delta B(z)=\frac{1}{24} B^{(4)}(0) z^{4}+\frac{1}{720} B^{(6)}(0) z^{6}+\ldots
$$

To determine the magnetic field according to the geometric center $(\mathrm{z}=0)$ of the coils is determined by the following expression:

$$
B(0)=\frac{16}{5 \sqrt{5}} \frac{1}{2} \frac{\mu_{0} N I}{R}
$$

\section{THE DESIGN AND SIMULATING OF HELMHOLTZ COILS}

The all of the design and simulating of coils was done in COMSOL Multiphysics software showed in Fig. 2.

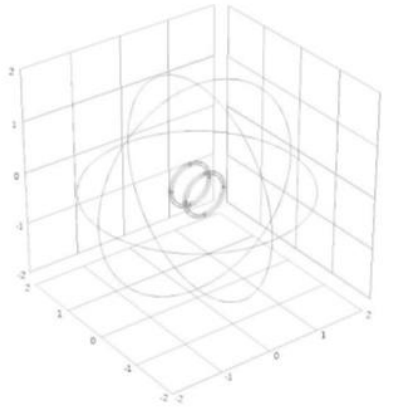

Fig.2. Geometry of Helmholtz coils in COMSOL

The simulating of the magnetic field produced by using module $\mathrm{mf}$ as only it allows you to use the section Multi-Turn Coil Domain for simulating this coil in 3D.

The grid size of coils was set $0.02 \mathrm{~m}$. The grid size of outer sphere was chosen Coarse

The two coils have a closed geometry with a circular crosssection perpendicular to the z-axis.

In addition, type of coil was choose of Circular type. The direction of the current in the coils corresponds to the geometric form of the coils, that is, along a circle in one direction in each of the coils in the direction of which our magnetic field is generated.

The Fig.3, 4 shows the generating finite element mesh.

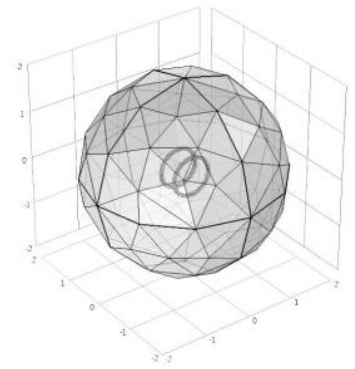

Fig.3. Generation the finite element mesh

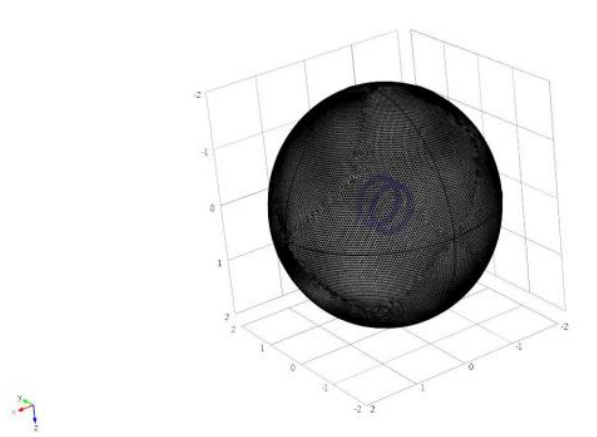

Fig.4. Generation the finite element mesh

After designing the Helmholtz coils in this program, we simulated our homogeneous field in the region of these coils.

The results showed the propagation and direction of the magnetic field vectors in the region of the generated field.

The simulation results of coils at a current power of $0.25 \mathrm{~mA}$ for the axial direction are showed in the Fig.5. 


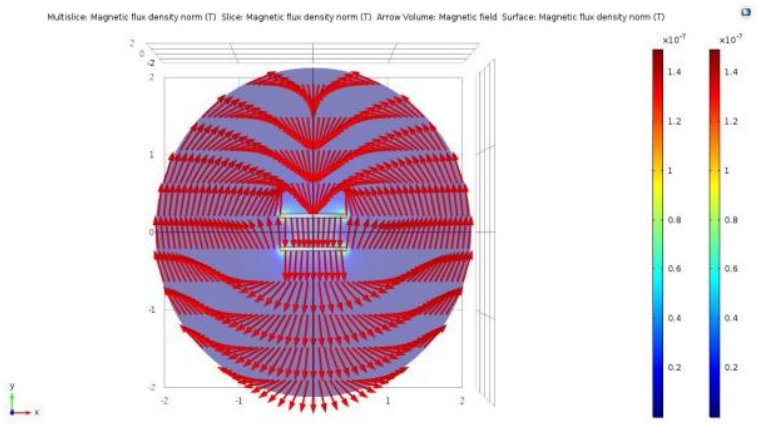

Fig.5. The distribution of the magnetic field in Helmholtz coils

As you can see in the Fig.6, the generated uniform field is concentrated in the central regions of the coils. The genetically determined field is measured in Tesla.

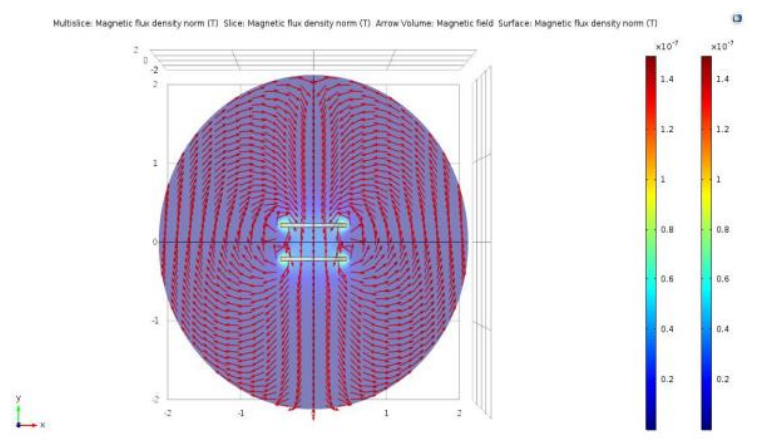

Fig.6. The distribution of the magnetic field in Helmholtz coils

The orientation of the vectors of the strengths of the generated uniform magnetic field is also illustrated in the Fig.7.

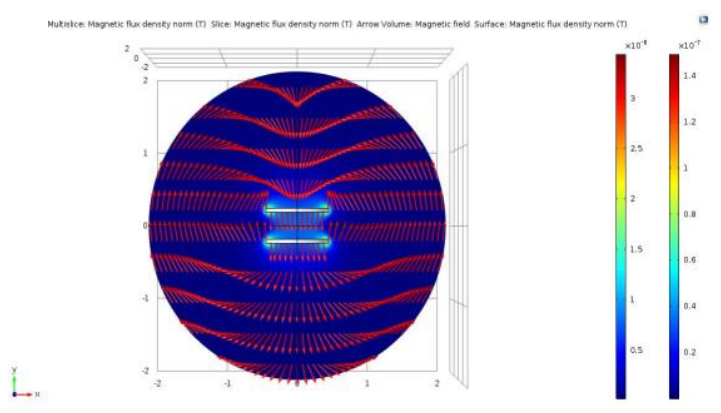

Fig.7. The distribution of the magnetic field in Helmholtz coils

Red and yellow-green colors are the places of maximum generation of a homogeneous field showed in Fig.8

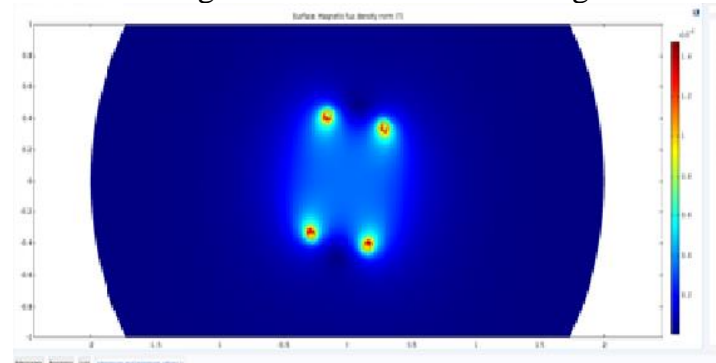

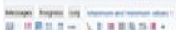

Fig.8. The areas of maximum generation of a magnetic homogeneous field

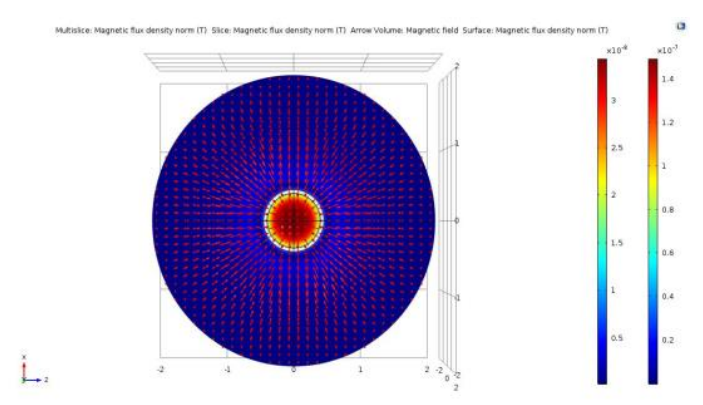

Fig.9. The distribution of the magnetic field in Helmholtz coils

\section{THE DESIGN OF SQUARE COILS}

Square coils can be utilized instead of circular coils in the design as they provide a wider uniform field parallel to the coils as compared to circular coils.

The governing equation for the magnetic field $B(z)$ can be calculated by formula:

$$
B(z)=\frac{\mu_{0} N I R^{2}}{\left.2\left(d^{2}+(z-d)^{2}\right)\right)^{2 / 3}}
$$

To simulate the magnetic field, a system of three pairs of square coils arranged usually perpendicular to one another (the Helmholtz cage) is used.

The sides of the squares of the coils are $2 \mathrm{~m}, 1,9 \mathrm{~m}, 1,8 \mathrm{~m}$. When a current flows through the coils in the region inside the cell, a uniform magnetic field is created, the direction and magnitude of which depend on the strength and direction of the current in the coils.

Below this a description to design a $3 \mathrm{D}$ model of cage we used SolidWorks software of the stand with the corresponding notation for the coils along the X-green, Y-red, Z-blue axes showed in Fig. 10.

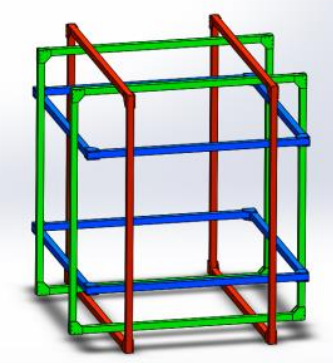

Fig. 10. 3D model of Helmholtz cage

The all of the design and simulating of coils was done in COMSOL Multiphysics software.

For our goals, we designed each pair of coils separately showed in Fig. 11. 


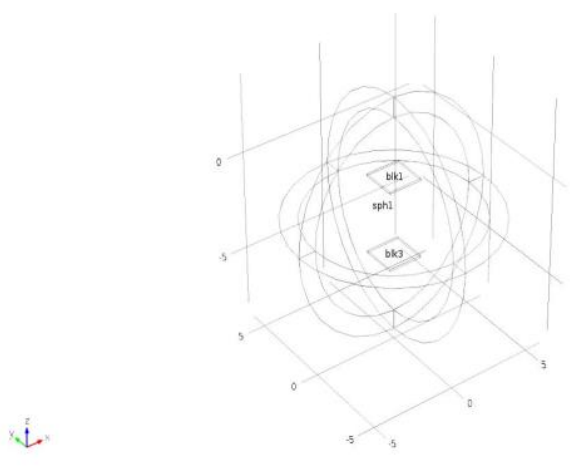

Fig.11. Geometry of square coil

The geometry of square coils showed in Fig. 12 .

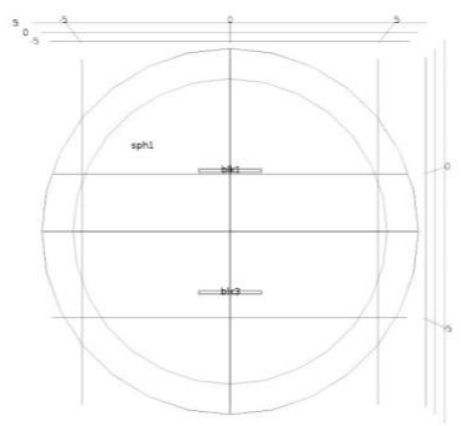

Fig.12. Geometry of square coils

In COMSOL Multiphysics, the $\mathrm{AC} / \mathrm{DC} \mathrm{mf}$ ('magnetic field') toolbox was used to simulate multi-turn coils with AWG12 wire (American Wire Gauge, Brown\&Sharpe), which we also used to construct the coil later on. The coils have a closed geometry with a circular cross section and each wire is insulated.

Around the coils, we created a sphere of air with a diameter of 6 meters and an infinite sphere around the air sphere with 1meter thickness (see Fig.12). The direction of current flow was specified by manually selecting a cross-sectional area of each coil, using the numeric coil type.

We used $1 \mathrm{~A}$ input current for the coils, which are connected in series.

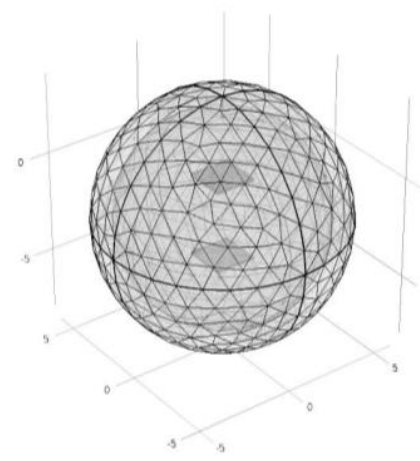

Fig.13. Output sphere with coils in finite mesh

The Fig.14 shows the external environment and coils without finite mesh

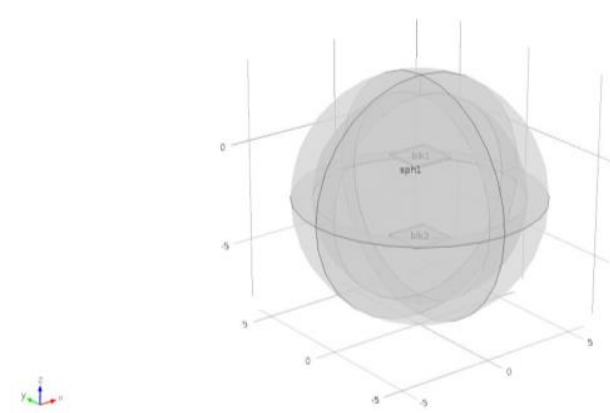

Fig.14. Output sphere with coils without finite mesh

After designing the Helmholtz coils in this program, we simulated our homogeneous field in the region of these coils.

The results showed the propagation and direction of the magnetic field vectors in the region of the generated field.

The simulation results of coils at a current power of $0.5 \mathrm{~A}$ for the axial direction are showed in the Fig. 15.

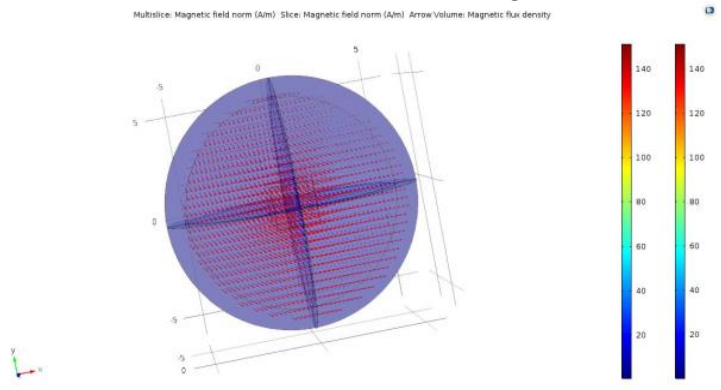

Fig.15. The distribution of the magnetic field in square coils

The orientation of the vectors of the strengths of the generated uniform magnetic field is also illustrated in the Fig.16

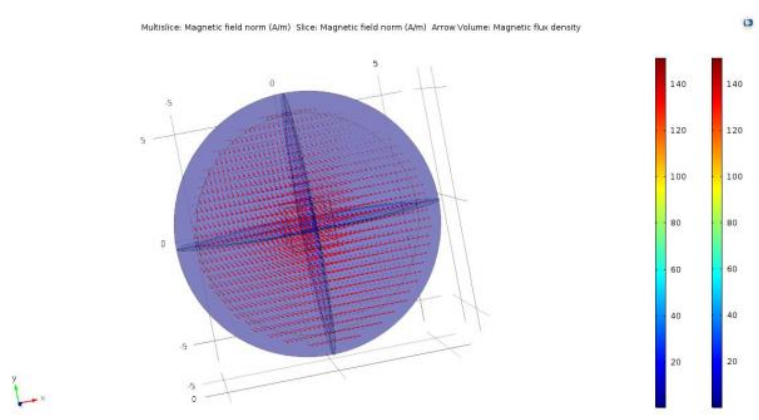

Fig.16. The distribution of the magnetic field in square coils

\section{CONCLUSION}

This article describes the basic theoretical and apathetic calculations of calculating Helmholtz coils to create a uniform magnetic field.

With models of circular coils, their geometric characteristics were modelled, 3D models of coils, models of the homogeneity of the magnetic field, and visualization of the direction of the stress vectors.

Based on these calculations given in this paper, it is entirely possible to create larger circular coils to create a stronger uniform magnetic field.

For designing Helmholtz coils of square type, theoretical and analytical models were analyzed, their geometric 
characteristics, 3D models of coils and visualization of the homogeneity of the magnetic field in these coils were modelled.

In future works, it is planned to correct the results in the design of the Helmholtz coils of square type. Recalculations of geometric characteristics, created homogeneity of the magnetic field, visualization of the orientation of the tension vectors in these coils will be done to realize a real model of these types of coils in assembly form.

It is also planned to use methods of amplification of a homogeneous magnetic field, taking into account the complexity of implementing their design as for a circular or square type, or in the arrangement of these two types of coils.

It is also possible to analyze the Helmholtz coils between existing coils, in order to compare the generated uniform magnetic field.

In addition, carrying out a comparative analysis of their geometric characteristics and reliability for creating real models.

\section{ACKNOWLEDGMENT}

This work has been supported financially by the research project №AP05132939 «Control system design of the satellite formation motion for remote sensing of the Earth», for 20182020 of the al-Farabi Kazakh National University, which is gratefully acknowledged by the authors.

\section{REFERENCES}

[1] Thesis Megan R. Brewer.Cubesat Attitude Determination and Helmholtz cage design.USA: Air force institute of technology, 19-20 (2012).

[2] Thesis R.A. Schill, and H. Karin "Characterizing and calibrating a large Helmholtz coil at low ac magnetic field levels with peak magnitudes below the earth's magnetic field," Review of Scientific Instruments, 2001, vol. 72, no 6, pp. 2769-2776. [2] Po Gyu Park, et al., "AC magnetic flux density standards in the low frequency range," in Proc. Conf. Dig. CPEM, June 2008, pp. 456-457. [3] Po Gyu Park, et al., "AC/DC magnetic flux density standard systems at KRISS," in Proc. Conf. Dig. CPEM, June 2010, pp. 312-313.

[3] Chapters J. C. Maxwell, "A Treatise on Electricity and Magnetism," Clarendon, Oxford, 1873, vol. I, Secs. 713, 714, and 715.

[4] Chapters D. Lorrain and D. R. Corson, "Electromagnetism," Freeman, San Francisco, 1979, Chap. 8.

[5] Chapters J. D. Kraus, "Electromagnetics," 2nd ed., McGraw-Hill, New York, 1973, Chap. 4

[6] Chapters P.M. Fishbane, S. Gasiorowicz, and S.T. Thornton, "Physics for Scientists and Engineers," 2nd ed., Prentice Hall, New York, 1996, Chap. 30.

[7] Thesis Vitalia E. Baranova, Pavel F. Baranov.Cubesat The Helmholtz Coils Simulating and Improved in COMSOL Russian Federation: National Research Tomsk Polytechnic University, 1-2 (2014).

\section{BIOGRAPHIES}

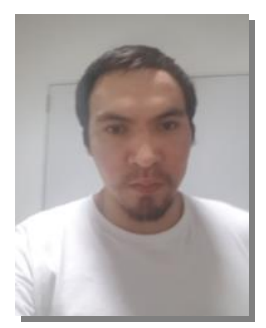

MUKHAMEDGALI ADIL Republic of Kazakhstan, Almaty City, in 1991. He received the B.S. degree in mechanical engineering and M.S. degree in space technic and technologies from the Kazakh National University named after Al-Farabi, Almaty city, B.S. in 2012 and M.S. in 2014.Since 2014, he has been assistant professor, senior teacher Department of the Department on Mechanics of alFarabi Kazakh National University. He is the author of 8 articles, and then 4 international conferences. His research interests include magnetic field modeling, nature of the geomagnetic fields, motion control and optimization, dynamics of nano- and microsatellites, applications in space engineering and technologies.

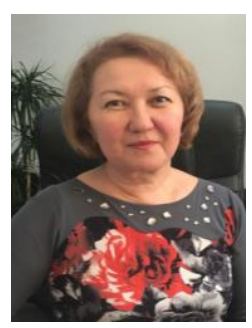

ZAURE RAKISHEVA Balkhash, Karaganda City, in 1964. She received the degree of specialist in mechanics from the M.V. Lomonosov Moscow State University, USSR, in 1986 and the Ph.D. degree in mechanics from Kazakh State University (now alFarabi KazNU), Almaty, Kazakhstan, in 1993.

From 1986 to 1996, she worked as engineer and researcher in the Scientific-Research Institutes of Academy of Sciences of Kazakhstan. Since 1996, she has been a Teacher, Associate Professor, Professor and Head of Department of the Department on Mechanics of al-Farabi Kazakh National University. She is the author of seven books, and more than 100 articles. Her research interests include dynamics of solid bodies, motion control and optimization, dynamics of nano- and microsatellites, applications in space engineering and technologies. 